\title{
The effect of antenatal corticosteroids on maternal glycemic control, in a tertiary care centre in North Kerala - India
}

\author{
Beena B. ${ }^{1 *}$, Libu G. K. ${ }^{2}$, Akhila M. S. ${ }^{1}$, Jyothi Chandran ${ }^{1}$
}

\begin{abstract}
${ }^{1}$ Department of Obstetrics and Gynecology, IMCH Medical College, Calicut, Kerala, India
${ }^{2}$ Department of Social and Preventive Medicine, Medical College, Trivandrum, Kerala, India
\end{abstract}

Received: 20 October 2019

Accepted: 05 November 2019

\author{
*Correspondence: \\ Dr. Beena B., \\ E-mail: aneebsdr@gmail.com
}

Copyright: (c) the author(s), publisher and licensee Medip Academy. This is an open-access article distributed under the terms of the Creative Commons Attribution Non-Commercial License, which permits unrestricted non-commercial use, distribution, and reproduction in any medium, provided the original work is properly cited.

\section{ABSTRACT}

Background: Antenatal glucocorticoid therapy (ACT) can be life-saving in preterm babies and risk of PTL is increasing in. The prevalence of GDM also increasing. Virtually, all women will experience deterioration in their glycaemic control following ACT. The NICE guideline recommends additional insulin and the national Indian guidelines recommend a $20 \%$ increase. Objective of this study was to evaluate the changes in maternal serum glucose following corticosteroids in antenatal women.

Methods: Prospective controlled trial of 206 patients, divided into 2 groups. Group 1:105 patients with no GDM and Group 2: 101 patients with GDM. Ethical committee approved. FBS and PPBS measured for following 5 days of D1. BMI and starting dose of insulin and dose of insulin hike were recorded. FBS $>90 \mathrm{mg} / \mathrm{dL}$ and PPBS $>120 \mathrm{mg} / \mathrm{dL}$ were taken as abnormal. Data were analyzed using SPSSV22.

Results: There were a total of 206 of which 105 were in group 1(51\%) and 101(49\%) were in Group 2 . FBS >90 $\mathrm{mg} / \mathrm{dL}$ in over $65 \%$ of all women on D2 10 new cases on D3 and PPBS $>120 \mathrm{mg} / \mathrm{dL}$ in over $66 \%$ of all women on D2 and 13 new cases on D3. Insulin was started in a total of 66 patients of $105(62.9 \%)$ in Group 1 and 33 out of 40 $(82.5 \%)$ in GDM on MNT. Of the 66 patients who started on insulin in Group 1, 17 (16.2\%) patients had to continue insulin and out of 33. In MNT subgroup $10(25 \%)$ had to continue. BMI was high in the patients, who was given and continued insulin.

Conclusions: The findings support the concept of increasing the dose of insulin even before the hyperglycemia documented. In the Group 166 of 105 patients were started on insulin due to hyperglycemia and 17 had to continue insulin 25.7\%), and 33 of 40 patients in GDM on MNT were started on insulin and 10 (30.33\%) had to continue. We suggest testing all patients for hyperglycemia and to start insulin especially in the those with high BMI (>25 as per the Asian Indian).

Keywords: Betamethasone, Body mass index, Corticosteroids, Fasting blood sugar, Gestational diabetes mellitus, Postprandial blood sugar, Preterm labor

\section{INTRODUCTION}

Antenatal glucocorticoid therapy can be life-saving in preterm babies and is commonly used in women at risk of preterm birth. Use of antenatal corticosteroid prophylaxis for threatened preterm labor has become more prevalent in recent years. Antenatal corticosteroid therapy (ACT) in which a potent synthetic glucocorticoid, betamethasone or dexamethasone, is administered to women at risk of preterm delivery is widely used to mature the fetal lungs. This aims to reduce neonatal morbidity and improve survival of preterm babies. ${ }^{1}$ The 1994 NIH consensus conference on antenatal corticosteroids and the American Congress of Obstetricians and Gynecologists have both recommended that women at risk to deliver infants at gestational ages of 
24 to 34 weeks be treated with corticosteroids in an attempt to accelerate fetal pulmonary maturity. ${ }^{2}$ The risk of preterm delivery is increased in women with PGDM, and the prevalence of PGDM is increasing. ${ }^{3}$ Thus, it is highly likely that physicians who care for pregnant women will encounter patients with diabetes receiving corticosteroids to enhance fetal pulmonary maturity.

Glucocorticoids are drugs, which have multiple effects on carbohydrate, protein, and lipid metabolism, as well as other aspects of homeostasis. They are also potent anti inflammatory and immunosuppressive molecules. One common side-effect of these drugs is their propensity to cause hyperglycemia. ${ }^{4}$ These drugs are used in pregnancy to accelerate lung maturity in the preterm fetus. Antenatal corticosteroids were first recommended for use in the National Institutes of Health (NIH) Consensus Development Conference statement 1995. Use of glucocorticoids, i.e., dexamethasone or betamethasone, in antenatal mothers at risk of preterm delivery, prevents respiratory distress and lowers the risk of hyaline membrane disease in their preterm infants. Glucocorticoids increase alveolar surfactant, improve pulmonary compliance. This occurs due to induction of protein synthesis in surfactant-producing type II cells in the fetal lung.

Gestational diabetes is not at all considered as a relative contraindication for ACT. In fact, women with gestational diabetes were more likely (odds ratio 1.21; $95 \%$ confidence interval 1.05-1.40) to receive ACS in a study conducted in British Columbia, Canada. ${ }^{5}$ Unfortunately, there are frequently maternal side effects from this therapy, the most concerning of which is the induction of hyperglycaemia due to increased insulin resistance. Steroid administration may induce clinically significant hyperglycaemia during pregnancy in women with or without gestational diabetes as well as in women with pre-existing type 1 or type 2 diabetes. Virtually all women with PGDM or GDM will experience deterioration in their glycemic control resulting in serum glucose levels above the range associated with optimal pregnancy outcome despite ongoing care and increases in insulin administration. An increase in insulin doses of at least $30-50 \%$ (average of $40 \%$ ) is usually required. ${ }^{6}$ Women with type 1 DM should have a urine ketone estimation during administration of corticosteroids.

The NICE guideline recommends that diabetic women receiving steroids should have additional insulin according to an agreed protocol. The national Indian guidelines on indoor management of diabetes recommend a $20 \%$ increase in the dose of insulin in persons with diabetes who receive steroid therapy. ${ }^{7}$

In the study by Ramirez-Torres, patients with GDM, treated by diet alone, required insulin, in $40 \%$ cases, which those already on insulin needed an increase of $39 \%$ to $112 \%$ in the daily insulin dose. An increased requirement of $26 \%$ to $64 \%$ was documented in women with pre-existing type 2 diabetes mellitus on insulin therapy. ${ }^{8}$

In a study by Allison et al, data generated demonstrate that in a large proportion of diabetic patients, despite efforts by clinicians with substantial experience caring for women with diabetes, suboptimal glucose control may continue for at longer period of time. Of the women who were in the hospital longer than 6 days, 34\% had suboptimal FSG and 30\% had suboptimal PPSG at the time of discharge or delivery. ${ }^{9}$ Betamethasone can also cause hyperglycemia in the non-diabetic obese patient. Non-diabetic women with obesity are at risk of hyperglycemia requiring insulin following betamethasone administration for fetal lung maturation. A relatively modest increase of insulin, if given early, may be sufficient to maintain glycemic control for most diabetic women. Increasing the dose of insulin earlier i.e. when the steroids are administered as opposed to waiting for glucose levels to deteriorate, may prevent the increase in serum glucose levels seen in virtually all women on days 2 and 3. Thus, the administration of corticosteroids may have long term hyperglycemic effects in women with previously diet controlled GDM.

\section{METHODS}

This study was done as a prospective trial in department of Obstetrics and Gynecology Government Medical College, Calicut, Kerala, during $1^{\text {st }}$ of August 2019 to $15^{\text {th }}$ of October 2019.Ethical committee clearance was obtained for the study.

All antenatal women who received corticosteroids for the enhancement of fetal pulmonary maturity during an inpatient admission were selected. They were divided into 2 groups.

\section{- Group 1: Women with no GDM/Pre GDM;}

- Group 2: Women with GDM on MNT, women with GDM on insulin/OHA and women with Pre GDM.

Patients with chronic kidney disease and those who required immediate termination of pregnancy were excluded. In Group 1, there were 104 patients and in Group 2, there were 102 patients.

Regular fetomaternal surveillance along with routine obstetric care was continued for all patients till discharge. NST was done on the days of betamethasone administration and on the day of discharge.

Data recorded included, maternal age, gestational age, indication for steroids, fasting and two-hour post prandial serum glucose levels, height and weight. Doses of oral anti-hyperglycemic agents and insulin given and urine ketone body in GDM on insulin and overt diabetic patients. Data collection began on the first day of steroid administration and continued for another 5 days. The day the first dose of corticosteroid was administered was 
designated day 1 (D1). Steroids were given on D1 and D2. The percentage of women with FBS $>90 \mathrm{mg} / \mathrm{dL}$ and the percentage of women who had PPBS $>120 \mathrm{mg} / \mathrm{dL}$ for the next five days after the initial dose of steroids were recorded.

\section{Statistical analysis}

Analysis was done using SPSS v 22 statistical package.

\section{RESULTS}

Total of 220 subjects were included in the study. As all the FBS and PPBS values (of 5 days) were not available for 14 patients, they were excluded from analysis. There was a total of 206 patients for final analysis; 105 were in group $1(51 \%)$ and $101(49 \%)$ were in Group 2. Both groups were comparable by their age and gestational age on the day steroid administration. In Group 2, 40 (19.4\%) patients were with GDM on MNT (medical nutritional therapy), $14(6.8 \%)$ patients belonged to GDM on OHA (oral hypoglycemic agents), 35 (17\%) were in GDM on insulin and $12(5.8 \%)$ were overt diabetic (Table 1).

Table 1: Study population.

\begin{tabular}{|lll|}
\hline Status & Frequency & Percent $(\%)$ \\
\hline Normal & 105 & 51 \\
\hline GDM on MNT & 40 & 19.4 \\
\hline GDM on OHA & 14 & 6.8 \\
\hline GDM on insulin & 35 & 17 \\
\hline PREGDM & 12 & 5.8 \\
\hline Total & $\mathbf{2 0 6}$ & $\mathbf{1 0 0}$ \\
\hline
\end{tabular}

Historical data provided on admission indicated that, Group 1 patients had a normal 75 gm GTT and a normal FBS/PPBS value prior to admission and the Group 2 patients were having good glycemic control with FBS below $90 \mathrm{mg} / \mathrm{dL}$ and PPBS below $120 \mathrm{mg} / \mathrm{dL}$.

Table 2: Number of patients with elevated FBS day 2 (D2), day 3 (D3), day 4 (D4), day 5 (D5), day 6 (D6) in the study population.

\begin{tabular}{|lllllll|}
\hline & D2 & D3 & D4 & D5 & D6 & Total \\
\hline Normal & 62 & 61 & 45 & 24 & 14 & 105 \\
\hline GDM on MNT & 30 & 28 & 23 & 12 & 3 & 40 \\
\hline GDM on OHA & 13 & 12 & 10 & 6 & 6 & 14 \\
\hline GDM on insulin & 20 & 29 & 24 & 17 & 12 & 35 \\
\hline PREGDM & 10 & 11 & 10 & 6 & 3 & 12 \\
\hline Total & & & & & & $\mathbf{2 0 6}$ \\
\hline
\end{tabular}

Table 3: Number of patients with elevated PPBS day 2 (D2), day 3 (D3), day 4 (D4), day 5 (D5), day 6 (D6).

\begin{tabular}{|lllllll|}
\hline & D2 & D3 & D4 & D5 & D6 & Total \\
\hline Normal & 62 & 56 & 39 & 22 & 16 & 105 \\
\hline GDM on MNT & 28 & 26 & 20 & 18 & 6 & 40 \\
\hline GDM on OHA & 11 & 12 & 10 & 8 & 7 & 14 \\
\hline GDM on insulin & 25 & 27 & 24 & 18 & 11 & 35 \\
\hline PREGDM & 11 & 12 & 10 & 8 & 2 & 12 \\
\hline Total & & & & & & 206 \\
\hline
\end{tabular}

FBS $>90 \mathrm{mg} / \mathrm{dL}$ seen in over $65 \%$ of all women on D2 $(135 / 206)$ and 10 new cases on D3 and PPBS >120 $\mathrm{mg} / \mathrm{dL}$ in over $66 \%$ of all women on $\mathrm{d} 2(137 / 206)$ and 13 new cases on D3. In Group 1, 62 patients out of 105 (59.6\%), showed elevated FBS on day 2 and $61(57.7 \%)$, showed elevated value on day 3 and the frequency declined on days 4, 5 and 6. PPBS values also showed a similar trend with 62 patients out of $105(59.6 \%)$ on day 2, 55 patients out of 104 (52.9\%) on D3 (Table 2 and 3).

The relationship between BMI and the starting of insulin and change in insulin dosage was analysed (Figure 1). In Group 1, those in whom insulin was not started (39 out of
105), had a lower BMI (37.1\%) than the patients in whom insulin was started (62.9\%), this was statistically significant. BMI of those in MNT subgroup also showed statistically significant relation with insulin was not started (39 out of 105), had a lower BMI (37.1\%) than the patients in whom insulin was started $(62.9 \%)$, this was statistically significant. BMI of those in MNT subgroup also showed statistically significant relation with the insulin.

Insulin was started in a total of 66 patients of 105 $(62.9 \%)$ in Group 1 and 33 out of $40(82.5 \%)$ in GDM on diet subgroup of Group 2. None of the patients in overt 
DM group had a positive urine ketone body test. BMI was analyzed on the basis of Asian Indian criteria (Figure 1). 71 patients out of $105(67.6 \%)$ in Group 1 was obese and $12(11.4 \%)$ were in the overweight category. In the GDM on diet subgroup, $27(67.5 \%)$ were obese and 7 $(17.5 \%)$ were overweight.

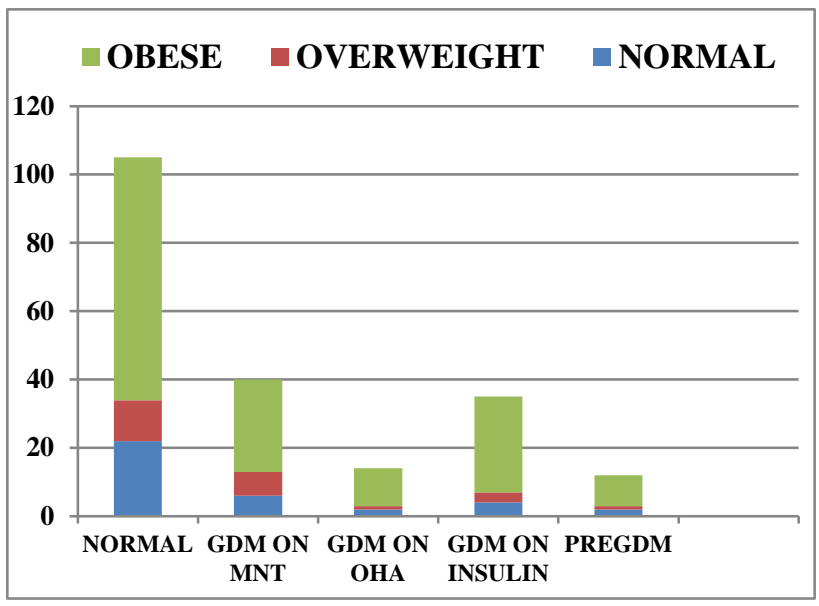

Figure 1: BMI (Asian Indian) in study group.

Table 4: Insulin with BMI in Group 1, p value is less than 0.05 .

\begin{tabular}{|lll|}
\hline & $\begin{array}{l}\text { No. of patients } \\
\text { (insulin started) }\end{array}$ & $\begin{array}{l}\text { Insulin } \\
\text { continued }\end{array}$ \\
\hline Normal BMI & 6 & 0 \\
\hline Overweight & 9 & $3(33.33 \%)$ \\
\hline Obese & 51 & $14(27.5 \%)$ \\
\hline
\end{tabular}

Table 5: Insulin with BMI (GDM on MNT), p value is less than 0.05 .

\begin{tabular}{|lll|}
\hline & $\begin{array}{l}\text { No. of patients } \\
\text { (insullin started) }\end{array}$ & $\begin{array}{l}\text { Insulin } \\
\text { continued }\end{array}$ \\
\hline Normal BMI & 4 & $1(25 \%)$ \\
\hline Overweight & 3 & $1(33.33 \%)$ \\
\hline Obese & 26 & $8(30.76 \%)$ \\
\hline
\end{tabular}

Insulin doses (those who were started newly on insulin and those who had increased doses) were also analyzed. Insulin was started in a total of 66 patients of 105 $(62.9 \%)$ in Group 1, and 33. Out of $40(82.5 \%)$ of GDM on MNT subgroup of Group 2. Of the 14 patients of GDM on OHA subgroup $10(71.4 \%)$ were started on insulin. Out of 35 patients who were in GDM on insulin group 23 patients received a higher dose of insulin and 11 continued with similar dosage and out of 12 in overt DM, 9 patients received a higher dose. Of the 66 patients who started on insulin in Group 1, $17(16.2 \%)$ patients had to continue insulin and out of 33. In MNT subgroup 10 (25\%) had to continue with insulin at the time of discharge after 6 days.
The data generated in this study showed 66 Women of 105 in Group 1 (majority started with a dose of 8 units, median dose is 8 and mean is 8.11), 33 women of 40 in GDM on MNT, subgroup ,majority were started on 8units with median 8 and mean 8.06) and 10 of 14 (GDM on OHA) was started on insulin, as they showed hyperglycemia following ACT.

In the GDM on insulin subgroup in 23 out of 35 , we have to increase the dose to an average of $47 \%$. In the overt DM subgroup, in 9 out of 12 patients we have increased the dose of insulin by an average of $64 \%$.

The relationship between BMI and the starting of insulin and change in insulin dosage was analysed (Table 4 and 5). In Group 1, those in whom insulin was not started (39 out of 105), had a lower BMI (37.1\%) than the patients in whom insulin was started $(62.9 \%)$, this was statistically significant. BMI of those in MNT subgroup also showed statistically significant relation with insulin.

\section{DISCUSSION}

The 1994 NIH Consensus and the American Congress of Obstetricians and Gynecologists have both recommended that women at risk of PTL at gestational ages of 24 to 34 weeks should be treated with corticosteroids. Moreover the risk of preterm delivery is increased in women with GDM, and the prevalence of GDM is increasing. ${ }^{10,11}$ Glucocorticoid administration induces profound insulin resistance mainly by its inhibition of glucose uptake and its reduction in the glucose storage pathway. ${ }^{12}$ The third trimester of pregnancy is characterized by insulin resistance with an increase in insulin need of $60 \%$ compared to pre-pregnancy level.

Previous studies in non-diabetic women, have suggested that the impact of corticosteroids on maternal blood glucose lasts 24 hours. $^{7}$ In our study, 66 out of 105 had an elevation of FBS and PPBS and they were started on insulin and 17 were Continued with insulin. This accounts for $25.76 \%$ of those who were started on insulin and these patients had a higher BMI. This indicates that, hyperglycemia lasts for more than 6 days in almost $25 \%$ of normal patients, who were obese.

In a study by Allison et al, data generated demonstrate that in a large proportion of diabetic patients, despite efforts by clinicians with substantial experience caring for women with diabetes, suboptimal glucose control may continue for at longer period of time. ${ }^{13}$ Of the women who were in the hospital longer than 6 days, 34\% had suboptimal FSG and $30 \%$ had suboptimal PPSG at the time of discharge or delivery. As in comparison, our data also showed a continued rise in FBS IN 13.5\% (14/105) of normal patients and $23.76 \%$ (24/101), in the GDM group. PPBS was high in $15.2 \%(16 / 105)$ and $25.74 \%$ (26/101) patients. 
We have reinforced MNT in all patients after receiving ACT, irrespective of their prior glycemic status. They were advised to avoid high calorie food for 5 days after ACT and to continue with that for those continued on insulin. Patients in Group 1 and GDM on MNT subgroup, who showed hyperglycemia were obese. Hence at least in patients with high BMI more stringent monitoring of blood sugar following ACT should status, especially in those who are obese and to continue MNT in them and to either to start insulin or to escalate the dose of insulin. Fetomaternal surveillance should be continued the dose of insulin even before the hyperglycemia is documented.

The findings support the concept of increasing be recommended. Those who started and continued on insulin among these patients also showed a significant association with obesity. Hence, strict monitoring of glycemic the dose of insulin even before the hyperglycemia documented. ${ }^{6}$ In the Group 1 (with prior euglycemia) 66 of 105 (62.9\%) patients were started on insulin due to hyperglycemia and 17 had to continue insulin $(25.76 \%$ ), and 33 of 40 patients in GDM on MNT $(82.5 \%)$ were stated on insulin and $10(30.30 \%)$ had to continue. Authors suggest testing all these patients for the hyperglycemia especially in the those with a high BMI (more than 25, as per the Asian Indian criteria) and start insulin.

The data generated in this study showed 66 Women of 105 in Group 1 (majority started with a dose of 8 units, median dose is 8 and mean is 8.11), 33 women of 40 in GDM on MNT, subgroup ,majority were started on 8units with median 8 and mean 8.06) and 10 of 14 (GDM on OHA) was started on insulin, as they showed hyperglycemia following ACT.

In the GDM on insulin subgroup in 23 out of 35 , we have to increase the dose to an average of $47 \%$. In the overt DM subgroup, in 9 out of 12 patients we have increased the dose of insulin by an average of $64 \%$. This is in accordance with Mathiesen ER, et al, where an average of $40 \%$ increase in insulin dose required. ${ }^{6}$

In Group 1, those in whom insulin was not started (39 out of 105), had a lower BMI $(37.1 \%)$ than the patients in whom insulin was started $(62.9 \%)$, this was statistically significant. BMI of those in MNT subgroup also showed statistically significant relation with the insulin dose.

\section{CONCLUSION}

The findings support the concept of increasing the dose of insulin even before the hyperglycemia documented. We suggest to test all patients for the hyperglycemia especially in the those with high BMI (> 25 as per the Asian Indian) and to start insulin accordingly.

Funding: No funding sources

Conflict of interest: None declared
Ethical approval: The study was approved by the Institutional Ethics Committee

\section{REFERENCES}

1. Liggins GC, Howie RN. A controlled trial of antepartum glucocorticoid treatment for prevention of the respiratory distress syndrome in premature infants. Pediatr. 1972;50:515-25.

2. Gilstrap LC, Christensen R, Clewell WH. National Institutes of Health (NIH). Effect of corticosteroids for fetal maturation on perinatal outcomes. NIH Consensus Statement. 1994;12:1-24.

3. Hanson U, Persson B. Outcome of pregnancies complicated by type 1 insulin-dependent diabetes in Sweden. Acute pregnancy complications, neonatal mortality and mortality. Am J Perinatol. 1993;10:330-3.

4. Landy JH, Isada N, McGinnis J, Ratner R, Grossman $\mathrm{JH}$. The effect of chronic steroid therapy on glucose tolerance in pregnancy. Am J Obstet Gynecol. 1988;159:612-5.

5. Kazem M, Hutcheon JA, Joseph KS. A populationbased study of antenatal corticosteroid prophylaxis for preterm birth. J Obstet Gynaecol Can. 2012;34:842-8.

6. Mathiesen ER, Christensen AB, Hellmuth E, Hornnes P, Stage E, Damm P. Insulin dose during glucocorticoid treatment for fetal lung maturation in diabetic pregnancy: Test of an algorithm. Acta Obstetricia et Gynecologica Scandinavica. 2002;81:835-9.

7. Bajwa SS, Baruah MP, Kalra S, Kapoor MC. Guidelines on Inpatient Management of Hyperglycemia. In: Muruganathan A, editor. Medicine Update. Vol. 23. Association of Physicians of India; 2013:164-169.

8. Ramírez-Torres MA, Pérez-Monter SE, Espino Y, Sosa S, Ibargüengoitia-Ochoa F. Effect of betamethasone in blood glucose levels in pregnant diabetic women at risk of preterm birth. Ginecol Obstet Mex. 2011;79:565-71.

9. Kreiner A, Gil K, Lavin J. The effect of antenatal corticosteroids on maternal serum glucose in women with diabetes; Open J Obstet Gynecol. 2012;2:112-5.

10. Melamed N, Chen R, Soiberman U, Ben-Haroush A, Hod M, Yogev, Y. Spontaneous and indicated preterm delivery in pregestational diabetes mellitus: Eti- ology and risk factors. Ach Gynecol Obstet. 2008;278:129-34.

11. Lawrence JM, Contreras R, Chen W, Sacks DA. Trends in the prevalence of pre-existing diabetes and gestational diabetes mellitus among a racially/ethnically diverse population of pregnant women, 1999-2005. Diabetes Care. 2008;31:899904.

12. Tappy L, Randin D, Vollenwieder P. Mechanisms of dex-induced insulin resistnce in healthy humans. J Clin Endocrinol Metab. 1994;79:1063-9. 
13. Foglia L, Deering S, Lim E, Landy H. Maternal glucose levels after dexamethasone for fetal lung development in twin vs singleton pregnancies. Am J Obstet Gynecol. 2008;199:380-1.
Cite this article as: Beena B, Libu GK, Akhila MS, Chandran JR. The effect of antenatal corticosteroids on maternal glycemic control, in a tertiary care centre in North Kerala - India. Int J Reprod Contracept Obstet Gynecol 2019;8:4668-73. 